\title{
A Comparison of Narrative Exposure Therapy, Supportive Counseling, and Psychoeducation for Treating Posttraumatic Stress Disorder in an African Refugee Settlement
}

\author{
Frank Neuner, Margarete Schauer, and \\ Christine Klaschik \\ University of Konstanz and Vivo
}

\author{
Unni Karunakara \\ Johns Hopkins University School of Public Health and Vivo
}

\author{
Thomas Elbert \\ University of Konstanz and Vivo
}

\begin{abstract}
Little is known about the usefulness of psychotherapeutic approaches for traumatized refugees who continue to live in dangerous conditions. Narrative exposure therapy (NET) is a short-term approach based on cognitive-behavioral therapy and testimony therapy. The efficacy of narrative exposure therapy was evaluated in a randomized controlled trial. Sudanese refugees living in a Ugandan refugee settlement $(N=43)$ who were diagnosed as suffering from posttraumatic stress disorder (PTSD) either received 4 sessions of NET, 4 sessions of supportive counseling (SC), or psychoeducation (PE) completed in 1 session. One year after treatment, only $29 \%$ of the NET participants but $79 \%$ of the SC group and $80 \%$ of the PE group still fulfilled PTSD criteria. These results indicate that NET is a promising approach for the treatment of PTSD for refugees living in unsafe conditions.
\end{abstract}

Experience of traumatic life events has been increasing in waraffected populations as civilians are increasingly exposed to war, torture, and forced migration. Refugees who have fled from war regions often report a number of extremely stressful experiences such as physical and psychological torture, shelling, sexual violence, and other atrocities. As a consequence, high prevalence of posttraumatic stress disorder (PTSD) has been reported in these populations (de Jong et al., 2001; Mollica et al., 1993; for a review, see Hollifield et al., 2002). This high number of traumatized survivors supports the notion that psychosocial services in refugee camps need workable guidelines on how to assist traumatized individuals in war-affected societies.

Within the past decade, empirical knowledge about effective psychotherapeutic treatment for PTSD has rapidly increased (Foa, 2000). Treatment for PTSD is well studied in civilian populations

Frank Neuner, Margarete Schauer, Christine Klaschik, and Thomas Elbert, Department of Clinical Psychology, University of Konstanz, Konstanz, Germany, and Vivo, Cupramontana, Italy; Unni Karunakara, Johns Hopkins University School of Public Health and Vivo.

Unni Karunakara is now at Médecins sans Frontières, Amsterdam, the Netherlands.

Research was funded by the Deutsche Forschungsgemeinschaft.

We thank Adi Gerstl from the German Developmental Service (DED), Mary A. Martin and Nicolas W. Krispo from the Sudanese Refugee Council, Elisabeth Kley and Elisabeth Schauer from Vivo, Cupramontana, Italy, for support in data collection and treatment, and Christina J. Robert for editing.

Correspondence concerning this article should be addressed to Frank Neuner, Department of Clinical Psychology, University of Konstanz, D-78457 Konstanz, Germany. E-mail: frank.neuner@uni-konstanz.de with victims of traumatic events who live in industrialized countries, such as the United States, Europe, or Australia. A large number of studies have proved that cognitive-behavioral therapy (CBT), especially exposure and cognitive techniques, are a powerful method of treating PTSD with those who experienced sexual assault (Foa et al., 1999; Foa, Rothbaum, Riggs, \& Murdock, 1991) as well as those recovering from physical assault or accidents (Marks, Lovell, Noshirvani, Livanou, \& Thrasher, 1998; Tarrier et al., 1999).

In contrast, the knowledge about effective treatment of PTSD in populations of civilians who have been affected by war is still scarce. Notwithstanding the considerable attention war-torn populations have received from psychosocial organizations in recent years, very little clinical research has been conducted on how to adequately support and treat these groups. The majority of survivors of war and torture are unable to safely escape their countries, forced instead to flee to insecure places within their home country or to adjacent regions that are often equally affected by war and terror. In addition to living with violence, many of these refugees are also living in poverty, dependent on humanitarian aid, and suffering from malnourishment.

These living conditions question the applicability of psychotherapeutic treatment approaches that have been developed for western PTSD populations. First, it is unclear how many refugees suffering from PTSD seek and accept aid that might provide relief. Maslow's hierarchy of needs (Maslow, 1943), for instance, claims that treatment for psychological problems can hardly be addressed as long as the basic needs of nutrition and safety are pressing. Furthermore, it is questionable if any psychological assistance can be successful given that treatment protocols for PTSD typically 
require the establishment of a safe and reassuring environment, conditions that are difficult to meet within the context of refugee camps and settlements. In addition, any psychotherapy work that will take place must be brief given large numbers of people and limited monetary resources. Any broad scale treatment program must be pragmatic and easy for local personnel to learn, even with little or no access to medical or psychological education or additional training. Consequently, the method must be adaptable to any cultural environment and easily implemented. The oral tradition is a common element among many cultures; thus narrative approaches seem ideally suited to cross-cultural applications.

Psychosocial organizations usually provide different forms of counseling approaches to assist traumatized refugees (Van der Veer, 1998). These types of treatment, often referred to as "trauma-counseling" or "cross-cultural counseling," encompass a large variety of approaches, such as problem-solving procedures (Amani Trust, 1997) as well as group discussions and individual assistance including variants of exposure treatment (World Health Organization / United Nations High Commissioner for Refugees [WHO/UNHCR], 1996). In most cases, the counseling procedures and duration of treatment are not rigorously standardized. The lack of standardization poses a serious restraint for the evaluation of counseling. Little knowledge about the efficacy of counseling in general exists (Bower et al., 2002), and as of yet there is no clinical trial that examined the efficacy of counseling approaches for traumatized refugees.

A common element in brief interventions for the assistance of large numbers of traumatized refugees is psychoeducation, thought to cause some relief for traumatized people by explaining the nature of the symptoms caused by traumatic experiences and by demonstrating that this response is normal. Psychoeducation has been used as an add-on element with various treatment approaches (Foa \& Rothbaum, 1998; WHO/UNHCR, 1996). Some therapists suggest using psychoeducation as an economical and effective large-scale intervention to reduce the suffering of populations affected by war.

Only a minority of organizations providing psychological assistance have tried to adapt scientific knowledge from PTSD research to the conditions of traumatized refugees. As an exception, Paunovic and Ost (2001) showed that exposure treatment is a promising treatment approach for refugees. They compared the efficacy of a 16- to 20-session exposure-based treatment with a combination of cognitive and exposure interventions of equal duration for 16 refugees who had fled to Sweden. Both treatments were similarly effective. A limitation of this study was the rather rigid inclusion criteria. Therapy was only offered to refugees who had a lasting Swedish residence permit. In addition, the treatment was conducted in Swedish, and thus the patients had to be relatively well adapted to the Swedish culture. These findings can be applied only to the minority of refugees who manage to flee to Western countries and to adapt to the new culture. Furthermore, there was no control group in this study that controlled for spontaneous remission and unspecific effects.

We developed narrative exposure therapy (Neuner, Schauer, Elbert, \& Roth, 2002) as a standardized short-term approach based on the principles of cognitive-behavioral exposure therapy by adapting the classical form of exposure therapy to meet the needs of traumatized survivors of war and torture. In exposure therapy, the patient is requested to repeatedly talk about the worst traumatic event in detail while re-experiencing all emotions associated with the event. In the process, the majority of patients undergo a habituation of the emotional response to the traumatic memory, which consequently leads to a remission of PTSD symptoms. As most of the victims of organized violence have experienced many traumatic events, it is often impossible to identify the worst event before treatment. To overcome this difficulty, we based our approach on testimony therapy, a method of therapy created by Lira and Weinstein (published under the pseudonyms Cienfuegos and Monelli, 1983) to treat traumatized survivors of the Pinochet regime in Chile and successfully applied in an uncontrolled trial to Bosnian refugees in the United States (Weine, Kulenovic, Pavkovic, \& Gibbons, 1998). Instead of defining a single event as a target in therapy, the patient constructs a narration about his whole life from birth up to the present situation while focusing on the detailed report of the traumatic experiences.

The focus of the narrative exposure therapy procedure is twofold. As with exposure therapy, one goal is to reduce the symptoms of PTSD by confronting the patient with the memories of the traumatic event. However, recent theories of PTSD and emotional processing suggest that the habituation of the emotional responses is only one mechanism to improvement of symptoms. Other theories suggest that the distortion of the explicit autobiographic memory about traumatic events leads to a fragmented narrative of the traumatic memories, which results in the maintenance of PTSD symptoms (Ehlers \& Clark, 2000). Thus, the reconstruction of autobiographic memory and a consistent narrative should be used in conjunction with exposure therapy. Narrative exposure therapy places a focus on both methods, that is, the habituation of emotional responding to reminders of the traumatic event and the construction of a detailed narrative of the event and its consequences.

This treatment study was carried out with Sudanese refugees who fled from the civil war to Northern Uganda. Currently about 200,000 Sudanese refugees live in Uganda; many of them have lived in the country for several years. In the setting of a refugee settlement, we compared the efficacy of three treatment conditions in a randomized controlled trial. One group received psychoeducation only, which was completed in one session. This group served as a control group to monitor the development of refugees who received only a minimal intervention beyond the diagnosis procedure. A second group received four sessions of supportive counseling in addition to psychoeducation. The lack of standardized procedures of trauma counseling prevented the inclusion of a group that was representative for the current standard of trauma counseling. Thus, the main purpose of the supportive counseling group was to control for nonspecific treatment effects. Finally, a third group was offered four sessions of narrative exposure therapy, which also included a psychoeducation component. Standard clinical instruments were applied for the examination of treatment effects immediately after therapy as well as 4 months and 1 year after treatment. We predicted a significant impact of the treatment condition on the development of the psychological status of the participants after treatment. Instruments of posttraumatic stress were the main outcome measures. In addition, we applied measures of comorbid anxiety and depression as well as psychological health. Specifically, we expected the participants in the narrative exposure therapy group to present with the best outcome at the 1-year follow-up. 
Method

\section{Setting}

This study followed a project that researched the demography of forced migration (Karunakara et al., in press; Schauer et al., 2001). The first step of this project was to investigate the impact of war and forced migration on levels of fertility, child mortality, violence, and stress in Sudanese and Ugandan populations in northwestern Uganda and southern Sudan. Following the wide-scale survey, the Imvepi settlement in northern Uganda was selected for the treatment group, as the refugees living there presented with the highest prevalence of PTSD (51\%). In Imvepi, most refugees had experienced multiple traumatic events in the Sudanese civil war before they fled to Uganda. However, northern Uganda was not a safe exile for the refugees as the settlements were threatened and attacked by Sudanese and Ugandan rebel armies. At the time of this study (2000-2001), living conditions were still scarce, with most refugees suffering from poor nutrition and poverty.

\section{Participants}

We randomly chose 77 participants from a list of respondents who had previously been randomly selected in a hut-to-hut procedure for the survey. Of these, $43(56 \%)$ presented with PTSD according to criteria from the Diagnostic and Statistical Manual of Mental Disorders (4th ed. [DSM-IV]; American Psychiatric Association, 1994). All of the participants were offered the opportunity to participate in this treatment study, as no respondent met the exclusion criteria of mental retardation or psychosis as demonstrated by a clinical examination. The objectives of randomized controlled trials and of this study in particular were fully explained to the patients. With the exception of only one respondent, all were willing to participate. Those who decided to participate gave written informed consent either with their own signature or the signature of a witness who was able to write. The informed consent and the study protocol were approved by the Ethical Review Board of the University of Konstanz and by the Ugandan National Council for Science and Technology, Kampala.
The respondents found it difficult to identify a single worst event, as in most cases they had experienced a series of traumatic events with similar high intensity. Instead of insisting on the identification of a single event, we asked for the most severe type of events they had experienced or witnessed. The majority of participants $(52 \%)$ reported the witnessing of people badly injured or killed as worst event type (which included the killing of relatives as well as massacres and mutilations); further worst event types were threats with weapons and kidnappings (17\%), physical attacks (12\%), torture (7\%), combat experiences (7\%), sexual assaults (5\%) and natural disasters $(2 \%)$. The average time past since the worst period of traumatic events was 7.5 years $(S D=3.3)$. Table 1 shows the sociodemographic characteristics of the participants in the treatment study. The randomization procedure resulted in different group sizes. There were no systematic group differences in any of the sociodemographic variables, as confirmed by Fisher's exact test and analysis of variance (ANOVA).

The participants received a compensation of 2,000 Ugandan shilling (equivalent to 1.25 U.S. dollars) for participation in the initial screening interview. They were told that they would receive no further monetary compensation or food for participation in the treatment study. However, to compensate for traveling to the location of the 4-month and 1-year follow-up examinations, we provided the equivalent of 3.00 U.S. dollars. Participants were not informed that they would be receiving this money when they agreed to participate and therefore did not expect any payment when participating in the treatment study or the follow-up interview.

\section{Local Team}

A team of nine research assistants was recruited from the refugee community. The assistants were selected from those interviewers who had already been employed in a previous large-scale epidemiological survey (Karunakara et al., in press; Schauer et al., 2001). They were in the age range of 20-51 years, had attended primary or secondary school, could write and read English, and had various occupational backgrounds (farmer, secretary, nurse assistant, teacher, etc.). In the epidemiological study, the research assistants had been trained in a 2-month course on principles of

Table 1

Sociodemographic Characteristics of Patients in the Different Treatment Groups

\begin{tabular}{|c|c|c|c|}
\hline Variable & $\begin{array}{l}\text { Narrative exposure therapy } \\
\qquad(n=17)\end{array}$ & $\begin{array}{l}\text { Supportive counseling } \\
\qquad(n=14)\end{array}$ & $\begin{array}{l}\text { Psychoeducation } \\
\quad(n=12)\end{array}$ \\
\hline Age in years $(S D)$ & $31.9(6.7)$ & $33.8(7.9)$ & $34.2(6.9)$ \\
\hline \multicolumn{4}{|l|}{ Sex: $n(\%)$} \\
\hline Male & $7 \quad(46.7)$ & $(42.9)$ & $(25.0)$ \\
\hline Female & $(53.3)$ & $8 \quad(57.1)$ & $9 \quad(75.0)$ \\
\hline \multicolumn{4}{|l|}{ Marital status: $n(\%)$} \\
\hline Single & $4 \quad(25.0)$ & $(14.3)$ & $1 \quad(8.3)$ \\
\hline Married/cohabiting & $12 \quad(75.0)$ & $10 \quad(71.4)$ & $8 \quad(66.7)$ \\
\hline Widowed & $0 \quad(0.0)$ & $2(14.3)$ & $3(25.0)$ \\
\hline \multicolumn{4}{|l|}{ Religion: $n(\%)$} \\
\hline Catholic & $(25.0)$ & $(35.7)$ & $5 \quad(41.7)$ \\
\hline Protestant/other Christian & $11 \quad(68.8)$ & $7 \quad(50.0)$ & $7 \quad(48.3)$ \\
\hline Other & $1 \quad(6.3)$ & $2(14.3)$ & $0 \quad(0.0)$ \\
\hline \multicolumn{4}{|l|}{ Education: $n(\%)$} \\
\hline None & $13 \quad(81.3)$ & $14 \quad(100.0)$ & 11 (91.6) \\
\hline Primary school & (18.7) & $0 \quad(0.0)$ & $1 \quad(8.4)$ \\
\hline \multicolumn{4}{|l|}{ Occupation: $n(\%)$} \\
\hline None & $3(18.8)$ & $5 \quad(35.7)$ & $4 \quad(33.3)$ \\
\hline Agriculture & $8(50.0)$ & $7 \quad(50.0)$ & $5 \quad(41.7)$ \\
\hline Other & $5 \quad(31.3)$ & $2 \quad(15.3)$ & $3 \quad(25.0)$ \\
\hline No. of possessions: $M(S D)$ & $1.4(0.6)$ & $1.5(1.2)$ & $1.9(1.2)$ \\
\hline No. of meals per day: $M(S D)$ & $1.3(0.6)$ & $1.4(0.2)$ & $1.6(0.8)$ \\
\hline Years since last migration: $M(S D)$ & $3.2(0.9)$ & $3.4(0.7)$ & $3.4(0.5)$ \\
\hline No. of traumatic event types: $M(S D)$ & $9.3(6.7)$ & $11.4(6.2)$ & $9.6(6.7)$ \\
\hline
\end{tabular}


quantitative data collection and interviewing techniques using the Demography of Forced Migration Questionnaire (DFMQ) and the Posttraumatic Stress Diagnostic Scale (PDS; both of which are described below). For the purpose of this treatment study, two of these research assistants (one male and one female) learned to apply additional instruments (the Self Reporting Questionnaire 20 [SRQ-20] and the 12-item version of the Medical Outcome Study Self Report Form [SF-12]; described below) and refreshed their abilities in the application of the DFMQ and the PDS in a 2-week course. Another six research assistants (two male and four female) were trained as translators for expert interviews and treatments using direct instructions and role plays. Except for the period of the 4-month follow-up, which was carried out by the interviewers alone, all research assistants were supervised at least once a week throughout the study, which included a review of the quality of their work and the discussion of possible emotional strain caused by interviews and translations.

\section{Instruments}

Expert interviews. The DSM-IV diagnosis and severity of PTSD was assessed with the PTSD portion of the Composite International Diagnostic Interview (CIDI; World Health Organization, 1997). The expert interviewers were doctoral-level psychologists or graduate students from Konstanz University who had been trained in PTSD assessment with the CIDI in previous field or laboratory studies. The items of the interview were translated before the interview and presented with the help of trained local translators. Further inquiries about details and examples of symptoms were made by the experts with the help of the translators to ensure a correct understanding of the symptoms and to validate the clinical significance of symptoms and the severity rating of PTSD.

Assisted self-report. The Demography of Forced Migration Questionnaire (DFMQ) was used to assess detailed information about demography, health, and traumatic experiences (for details see Karunakara et al., in press; Schauer et al., 2001). For the assessment of traumatic experiences, a checklist of 31 different types of traumatic events was administered. Each event was scored as present or not present in the respondent's life. The number of different experienced and witnessed types of traumatic events was used for the estimation of the severity of trauma exposure. The economical status was estimated from a household asset list that included essential items in a household such as blankets, pots, and so forth. The relevant indicator was the number of possessions per adult in a household. Nutrition was evaluated by asking for the number of meals eaten the previous day. Self rating of symptoms and functioning was carried out with the use of standardized clinical instruments. Because of a high illiteracy rate, the translations of the self-rating instruments were administered in the form of standardized interviews by a trained local team. The frequency and severity of PTSD symptoms was assessed with the PDS (Foa, 1995), modified in the translation procedure to simplify the frequency rating of symptoms. The Self-Reporting Questionnaire 20 (SRQ-20; Harding et al., 1980) was used to indicate comorbid symptoms of anxiety and depression. This instrument had been widely used in African settings previously. Psychological functioning was measured with the psychological health scale from the 12-item version of the Medical Outcome Study Self-Report Form (SF-12; Ware, Kosinski, \& Keller, 1996). This questionnaire was used to assess health-related quality of life in different cultures. Several items of the questionnaire had to be adapted to the living conditions of an African refugee settlement. As no comparison data were available for the Sudanese refugees, the usual standardization procedure was not used for scoring.

All instruments were translated into the Arabic dialect spoken by the refugees in Imvepi (Juba-Arabic). The questionnaires were translated using several steps of translations, blind back translations, and subsequent corrections by independent groups of translators who were recruited from the refugee community.

\section{Procedure}

Each respondent received two initial interviews in a screening phase, one by a local interviewer (DFMQ, PDS, SRQ-20, SF-12) and one by experts from Konstanz University (CIDI). Those who presented with PTSD in the CIDI interview were offered participation in the treatment study. Each participant was randomly assigned (using a dice) to one of three treatment groups: narrative exposure therapy, supportive counseling, or psychoeducation only. The treatment conditions consisted of one (psychoeducation) or four (narrative exposure therapy, supportive counseling) sessions of treatment. The first session started immediately after the initial expert interview. For the narrative exposure therapy and supportive counseling groups, the subsequent three treatment sessions were scheduled within the following 2 weeks. The duration of a treatment session was $90 \mathrm{~min}$ but could extend up to $120 \mathrm{~min}$ for exceptional sessions. Three to four weeks after the first interview, a posttest consisting of the standard battery of PDS, SRQ-20, and SF-12 measures was carried out by local interviewers. This battery was also administered at follow-up examinations four and 12 months after treatment. The 1-year follow-up test included a further interview with the CIDI administered by trained researchers. In addition, the trauma event checklist was applied once again to record the individual's history of traumatic events during the follow-up period.

The local and expert interviewers who carried out the posttests, as well as the follow-up tests, were blind for the individual participant's treatment condition. The respondents were instructed not to inform the interviewers or the trained researchers about the type of treatment or the number of sessions they had received. The initial interviews and the treatment sessions took place in huts or under trees around the settlement's medical center, which consisted of three straw huts. The posttest and follow-up test were carried out at the same place or in the respondents' huts for those who had moved to places that were too far away to travel to the settlement.

\section{Treatment}

Treatment was carried out by three female and two male therapists from the University of Konstanz and the aid organization Vivo (www.vivo.org) with the help of interpreters. Therapists were doctoral-level psychologists or graduate students with education and experiences in other treatment approaches including counseling. Therapists were trained in narrative exposure therapy and supportive counseling by the first and second authors-who had developed narrative exposure therapy and were educated in counseling - through direct observation of treatments. The allocation of translators to therapists could change over the course of the trial, but the single sessions of individual treatments were always carried out by the same pairs of therapists and translators. To rule out a possible therapist effect, each therapist provided approximately the same number of treatments in all conditions. Weekly meetings were scheduled to supervise the quality of treatments. As audio recordings were not practicable in the setting, treatment adherence was monitored by requesting the therapists to give a detailed account of either the participant's current life problems (supportive counseling) or the participant's life history recorded thus far (narrative exposure therapy) at least once for each treatment in the supervision meeting. In addition, selected treatment sessions were directly observed by the first or second author. No major deviations from treatment protocol were detected.

The first session always included psychoeducation about the nature and prevalence of PTSD symptoms independent of the treatment group. A standard written rationale was developed for this purpose. The goal of the procedure was to explain that PTSD-related symptoms and dysfunction frequently occur after multiple traumatic experiences. For the narrative exposure therapy and supportive counseling groups, psychoeducation was followed by the explanation of the respective treatment rationale. For participants in the psychoeducation group, no further treatment was offered. 
Narrative exposure therapy. In narrative exposure therapy, the participant constructs a detailed chronological account of his own biography in cooperation with the therapist. The autobiography is recorded by the therapist and corrected with each subsequent reading. A special focus of the therapy is on the transformation of the generally fragmented report of traumatic experiences into a coherent narrative. During the discussion of traumatic experiences, the therapist asks for current emotional, physiological, cognitive, and behavioral reactions and probes for respective observations. The participant is encouraged to relive these emotions while reporting the events. The discussion of a traumatic event is not terminated until a habituation of the emotional reactions presented and reported by the patient takes place. In the last session, the participant receives a written report of his biography (for further details of narrative exposure therapy, see Schauer, Neuner, \& Elbert, in press).

Supportive counseling. On considering the variety of mainly nonstandardized approaches currently applied in the field, we felt it was not possible to determine a supportive counseling procedure that was representative for the current standard. We applied a procedure that was flexible and open to modification depending on the participants' specific needs. The main goal of supportive counseling was to explore and strengthen the participants' individual, social, and cultural resources. The focus of the treatment was on current interpersonal problems, personal decisions, and plans and hopes for the future. To maintain a minimal level of standardization, a problem-solving approach, specifically developed for trauma counseling in Africa (Amani Trust, 1997), was generally used. To distinguish supportive counseling from narrative exposure therapy, we established a basic restriction of supportive counseling to exclude talk about specific past traumatic events, a goal that was achieved easily given the severity of the symptoms and the high level of avoidance in these patients. This restriction was especially useful as some representatives of psychosocial organizations reject talking about traumatic events within short-term treatment approaches given concerns about "retraumatization."

\section{Results}

\section{Participation}

As a consequence of the randomization procedure, 17 participants were offered narrative exposure therapy, 14 were offered supportive counseling, and 12 were offered psychoeducation. In the narrative exposure therapy group 1 patient refused to participate; all other patients agreed. Only in the supportive counseling group did any patients fail to complete the full treatment; 2 patients in this group discontinued treatment. The reason for not participating or for terminating treatment was generally due to a lack of time given the need to cultivate the fields at the beginning of the rainy season.

\section{Symptom Scores}

Table 2 presents the results of the symptom and health scales for pretreatment, posttreatment, 4-month follow-up, and 1-year follow-up time points. All participants who were randomized to treatment, including the 1 who refused participation in the narrative exposure therapy group and the 2 dropouts in the supportive counseling group, were included in the analyses.

As the randomization procedure resulted in different baseline levels for some measures, repeated measures ANOVAs were calculated with time as a four-level within-subject variable and treatment group as a three-level between-subjects variable for each outcome measure. Significant interactions between time and treatment were considered as the relevant indicators of a different development of the treatment groups over time. Mauchly's tests were calculated to test for the sphericity assumption of the univariate ANOVA. The sphericity assumption was met for the SRQ, the SF-12, and the CIDI, but not for the PDS. As the assumption of a homogeneous variance-covariance matrix inherent in a multivariate approach was met for the PDS (verified with Box's $M$ test), a multivariate analysis of variance instead of a univariate approach was calculated for this measure. To maximize use of information in this study with a small sample size, missing data were estimated with a restricted maximum likelihood procedure. To control for a possible confounding effect of further traumatic life events after treatment, the number of traumatic events reported for the 1-year follow-up period was entered as a covariate.

Differences between the treatment groups were examined with two contrast analyses for each outcome measure. In each of these analyses, we compared narrative exposure therapy with each other treatment condition with regard to the changes in the mean of the individual outcome variable between the pretest and the 1-year follow-up. As two calculations were carried out for each measure, significance level was Bonferroni corrected to an $\alpha$ of .025 for the contrast analyses.

A significant Time $\times$ Treatment interaction was found for the PDS (Wilks's $\lambda=.78$ ), $F(6,54)=4.30, p=.01, \eta^{2}=.31$. The contrast analysis revealed that the narrative exposure therapy group had a better improvement at the 1-year follow-up than did the supportive counseling group, $F(1,106)=5.65, p<.01$, as well as the psychoeducation group, $F(1,106)=14.00, p<.01$. This pattern was confirmed by the CIDI, with a significant Time $\times$ Treatment interaction, $F(2,34)=4.87, p=.01, \eta^{2}=.21$. In the contrast analysis, the narrative exposure therapy group differed significantly from the supportive counseling group, $F(1,34)=$ $7.05, p=.01$ as well as the psychoeducation group, $F(1,34)=$ $7.03, p=.01$. Contrary to our hypotheses, no significant Time $\times$ Treatment interaction was found for the SRQ, $F(6,106)=0.20$, $\eta^{2}=0.02, p>.90$, or for the SF-12, $F(6,106)=1.80, \eta^{2}=0.11$, $p=.10$. Contrast analyses showed a significant difference between narrative exposure therapy and supportive counseling for the SF-12, $F(1,106)=7.20, p<.01$, but not between narrative exposure therapy and psychoeducation, $F(1,106)=0.34, p=.54$. For the SRQ, neither the comparison between narrative exposure therapy and supportive counseling nor the comparison between narrative exposure therapy and psychoeducation revealed a significant difference.

\section{Clinical Significance}

Effect sizes (see Table 2) at posttest were calculated as the change of the mean between pre- and posttest divided by the pooled standard deviation of the outcome variable at pre- and posttest. Effect sizes at 1-year follow-up resulted from the analogous comparison between the means at pretest and the 1-year follow-up.

The percentage of patients with a diagnosis of PTSD for those patients who participated in the 1-year follow-up was compared for the three treatment groups. Table 3 presents the results of this analysis, indicating that only in the narrative exposure therapy group did the majority of participants exhibit an absence of PTSD 1 year after treatment. The differences between the treatment groups were significant, $\chi^{2}(2, N=38)=9.48, p<.01$. Differ- 
Table 2

Means (and Standard Deviations) and Effect Sizes of the Outcome Variables by Treatment Groups

\begin{tabular}{|c|c|c|c|c|c|c|c|c|}
\hline Treatment groups & & Pre & & Post & 4-month & 1-year & $\begin{array}{l}\text { Effect size } \\
\text { Pre to post }\end{array}$ & $\begin{array}{l}\text { Effect size } \\
\text { Pre to 1-year } \\
\text { follow-up }\end{array}$ \\
\hline \multicolumn{9}{|c|}{ Sample size } \\
\hline NET & & 17 & & 15 & 15 & 14 & & \\
\hline $\mathrm{SC}$ & & 14 & & 13 & 13 & 13 & & \\
\hline PE & & 12 & & 12 & 12 & 11 & & \\
\hline \multicolumn{9}{|c|}{ Posttraumatic Stress Diagnostic Scale } \\
\hline NET & 25.2 & (7.4) & 19.1 & (11.7) & $24.5 \quad(7.8)$ & 16.0 & 0.6 & 1.6 \\
\hline $\mathrm{SC}$ & 22.0 & $(8.0)$ & 19.8 & (10.9) & $22.8 \quad(10.1)$ & 23.1 & 0.2 & -0.1 \\
\hline PE & 19.5 & $(8.0)$ & 21.2 & $(9.4)$ & 27.7 (6.6) & $23.9 \quad(7.0)$ & -0.5 & -0.9 \\
\hline
\end{tabular}

Composite International Diagnostic Interview_PTSD part

\begin{tabular}{|c|c|c|c|}
\hline NET & $13.4 \quad(2.1)$ & $8.9 \quad(2.7)$ & 1.9 \\
\hline $\mathrm{SC}$ & $13.9 \quad(2.3)$ & $12.6(3.2)$ & 0.4 \\
\hline PE & $14.2 \quad(2.9)$ & $13.4(3.3)$ & 0.3 \\
\hline
\end{tabular}

Self-Reporting Questionnaire-20 score

\begin{tabular}{|c|c|c|c|c|c|c|c|c|c|c|}
\hline NET & 15.6 & (2.9) & 13.1 & (5.1) & 11.9 & (4.9) & 11.0 & (5.1) & 0.6 & 1.1 \\
\hline $\mathrm{SC}$ & 16.5 & (2.7) & 14.3 & $(5.0)$ & 12.8 & (3.9) & 12.4 & (4.8) & 0.5 & 1.0 \\
\hline PE & 18.6 & $(2.0)$ & 15.3 & (3.2) & 15.1 & (2.6) & 14.4 & (4.1) & 1.2 & 1.3 \\
\hline
\end{tabular}

Medical Outcome Study—Short Form 12-Psychological Health subscale

\begin{tabular}{lllllrr} 
NET & $0.27(0.12)$ & $0.36(0.19)$ & $0.38(0.12)$ & $0.44(0.19)$ & -0.6 & -1.1 \\
SC & $0.34(0.11)$ & $0.33(0.21)$ & $0.33(0.14)$ & $0.36(0.14)$ & 0.1 & 0.1 \\
PE & $0.23(0.15)$ & $0.33(0.19)$ & $0.37(0.14)$ & $0.35(0.17)$ & -0.7 & -0.8 \\
\hline
\end{tabular}

Note. The Composite International Diagnostic Interview was not assessed at posttreatment or at the 3-month follow-up. Pre $=$ pretreatment; post $=$ posttreatment; $\mathrm{NET}=$ narrative exposure therapy; $\mathrm{SC}=$ supportive counseling; $\mathrm{PE}=$ psychoeducation.

ences between single treatment groups were analyzed with Fisher's exact tests (Bonferroni corrected $\alpha=.025$ ). They showed that significantly fewer participants in the narrative exposure therapy group presented with PTSD than in the supportive counseling group, $p=.01$ or in the psychoeducation group, $p=.02$.

The SRQ-20 allows the classification of a patient as being a likely severe mental health case. The cut-off for this decision is a score of 10 or above. Table 4 shows the percentage of persons in each group who were classified as likely severe cases at the four time points. According to this index, before treatment all partici-

Table 3

Number and Percentage of Patients With a Diagnosis of Posttraumatic Stress Disorder (PTSD) According to the Composite International Diagnostic Interview at 1-Year Follow-Up

\begin{tabular}{lcc}
\hline \multicolumn{1}{c}{ Treatment } & $n$ & $\begin{array}{c}\text { PTSD at 1-year follow-up } \\
n(\%)\end{array}$ \\
\hline Narrative exposure therapy & 14 & $4(29)$ \\
Supportive counseling & 14 & $11(79)$ \\
Psychoeducation & 10 & $8(80)$ \\
\hline
\end{tabular}

pants were classified as suffering from severe mental problems. The difference in the distribution of cases and non-cases in the treatment group did not reach significance (Fisher's exact test $p=$ -.08 ) at 1-year follow-up.

At the 1-year follow-up assessment we observed that a large proportion of the refugees had left the settlement. We set up the post hoc hypothesis that the migration pattern depends on treatment and that more refugees from the narrative exposure therapy group had left the settlement. In the narrative exposure therapy

Table 4

Percentage of Patients Classified as Mental Health Cases According to Self-Reporting Questionnaire-20 Cutoff

\begin{tabular}{lcccc}
\hline Treatment & $\begin{array}{c}\text { Pretreatment } \\
(\%)\end{array}$ & $\begin{array}{c}\text { Posttreatment } \\
(\%)\end{array}$ & $\begin{array}{c}\text { 4-month } \\
\text { follow-up } \\
(\%)\end{array}$ & $\begin{array}{c}\text { 1-year } \\
\text { follow-up } \\
(\%)\end{array}$ \\
\hline $\begin{array}{c}\text { Narrative exposure } \\
\text { therapy }\end{array}$ & 100 & 73 & 73 & 50 \\
$\begin{array}{c}\text { Supportive } \\
\text { counseling }\end{array}$ & 100 & 85 & 85 & 77 \\
$\begin{array}{l}\text { Psychoeducation } \\
\text { Psychos }\end{array}$ & 100 & 92 & 100 & 91 \\
\hline
\end{tabular}


group, $62 \%$ had left the settlement, whereas this was the case in only $7 \%$ (one) of the supportive counseling group and 17\% (two patients) of the psychoeducation group. The difference was significant, $\chi^{2}(2, N=40)=12.34, p<.01$. Fisher's exact tests (Bonferroni corrected $\alpha=.025$ ) showed that from the narrative exposure therapy group significantly more refugees left than from the supportive counseling group $(p<.01)$ or from the psychoeducation group $(p=.01)$.

The incidence of one or more further traumatic events in the 1-year follow-up period was 93\%. The participants reported a mean of $3.55(S D=2.36)$ event types for the time between treatment and the examination. The mean number of traumatic event types differed significantly between those refugees who stayed $(M=4.00, S D=2.40)$ and those who left $(M=2.50, S D=$ 1.97) the settlement, $t(39)=2.20, p=.03$.

\section{Discussion}

We carried out a randomized controlled trial of three different short-term treatment methods for survivors of war experiences who suffered from PTSD in a settlement for Sudanese refugees in North Uganda. All but one of the participants who presented with PTSD in the screening interview agreed to participate in treatment, and only in the supportive counseling condition did two patients prematurely terminate treatment. This confirms that even in the desperate living conditions of a refugee settlement, without monetary or food assistance, people who suffer from PTSD seek psychosocial assistance. Although hope was one motivator, curiosity concerning the possibility to talk to White researchers also may have played a role in the high rate of acceptance. The fact that there was no dropout in the narrative exposure therapy group is noteworthy, as other treatment studies have reported high dropout rates for exposure therapy for PTSD of up to 28\% (Foa et al., 1991). One main difference between those studies and this one is the small number of treatment sessions for narrative exposure therapy (4), whereas other studies typically ranged from 9-12 sessions and included additional homework tasks. Furthermore, a unique motivator of narrative exposure therapy is the anticipation of receiving a written biography upon completion. Anecdotally we can report that participants stated that they were looking forward to the document even if they could not understand English text. Many patients hoped to pass on their biography to their children once they were living in peace and their children were educated. They wanted the next generation to be informed about their suffering during the war and the liberation fights. In addition, they encouraged us to publish the testimonies to educate the world about their situation in a war that has been forgotten by the developed world.

We observed different developments of the participants' symptoms after treatment. The PDS scores showed a worsening of PTSD symptoms for all groups between the posttest and the 4-month follow-up, possibly reflecting an increase in the occupation with the traumatic past, as food rations were cut down dramatically in this period and the refugees were under pressure to leave the settlement to places where they had experienced traumatic events.

At 1-year follow-up, the narrative exposure therapy group presented with a significantly better outcome on measures of posttraumatic stress than either of the other groups. This shows that the improvement of the narrative exposure therapy participants cannot be attributed to spontaneous recovery, as the psychoeducation participants who received a minimal intervention did not improve in the same way. Likewise, nonspecific treatment effects, which are quite probable in the confrontation with European therapists, cannot fully account for the efficacy of narrative exposure therapy, as the supportive counseling group did not benefit to the same extent. Despite the specific improvement of the narrative exposure therapy participants, the symptom scores still remained high but the change was clinically significant, as $71 \%$ of the narrative exposure therapy patients no longer fulfilled the DSM-IV criteria for PTSD, a significantly larger proportion than in the two other groups.

The size of the treatment effect on posttraumatic symptoms at posttest (0.6) was lower than the effect sizes reported in other treatment studies involving exposure techniques with traumatized populations in industrialized countries (e.g., Foa et al, 1999; Tarrier et al., 1999). However, when interpreting this value the fact that the psychoeducation group presented with a negative effect of -0.5 at posttest must be accounted for. This result indicated a worsening of those who received minimal intervention during the same time period. At the 1-year follow-up, the narrative exposure therapy effect sizes (1.6 for PDS and 1.9 for CIDI) indicated a clinically significant change for PTSD symptoms that did not occur in the other groups. These results indicate that the wellestablished knowledge about the efficacy of exposure techniques for the treatment of PTSD (Foa, 2000) can be transferred to refugee populations living in unsafe conditions.

All groups improved somewhat during the 1-year period after therapy, according to indicators of anxiety, depression, and overall mental health. However, the majority of participants were still classified as severe mental health case in the supportive counseling $(77 \%)$ and psychoeducation $(91 \%)$ groups. The narrative exposure therapy group showed more improvement than the other groups, yet the difference was not significant, as $50 \%$ of this group was still classified as severely psychologically disturbed. In addition, effect sizes did not indicate a superiority of the narrative exposure therapy group on these measures. This outcome possibly reflects the limitations of short-term psychosocial interventions for traumatized refugees who continue to remain in desperate living conditions. Factors such as insecurity, poverty, malnutrition, lack of medical assistance, and the hopeless political situation of the Sudanese refugees may restrict the impact of any psychosocial intervention.

Nevertheless, observation of the refugees' migration that occurred after treatment indicates that the narrative exposure therapy may have had an astonishing effect on decisions to leave the settlement. Approximately 2 months after the last treatments were completed a dramatic reduction of the food rations provided by UNHCR in Imvepi was implemented, resulting in the departure of many refugees from the camp. Refugees who left the settlement often moved to more safe and fertile places in self-settlements closer to the Sudanese border, moved back to Sudan, or sought jobs in the surrounding region. The majority of the participants in the narrative exposure therapy group managed to leave the settlement within 1 year following treatment, a tendency that did not occur for refugees in the other treatment groups. The comparison of the number of traumatic event types reported by the refugees for the 1 year period after treatment indicated that although almost all participants $(93 \%)$ reported the witnessing or experiencing of one 
or more further traumatic events, those who left the settlement did indeed move to safer places and reported a significantly lower number of event types. A reduction of avoidance symptoms caused by narrative exposure therapy might have empowered these refugees to move to areas that were closer to the war regions and to abandon the insufficient assistance and putative security provided in Imvepi. This post hoc finding may indicate a relationship between posttraumatic stress, treatment, and migration patterns that should be examined with specific hypotheses in further studies.

To our knowledge, this is the first randomized controlled trial of treatment for traumatized survivors of war living in a developing country. The study shows that refugees who suffer from PTSD seek and accept psychosocial treatment even when living in difficult living conditions. Narrative exposure therapy was superior to counseling and psychoeducation with regard to significant longterm changes in PTSD symptoms and behavior. These changes even had direct effects on the life of the refugees. Unfortunately, comorbid symptoms were not sufficiently improved in the majority of patients as a result of treatment.

The lack of a long-term effect on both PTSD and comorbid anxiety and depression for the supportive counseling condition brings into serious question the current practice of many psychosocial organizations to apply untested counseling approaches as a standard method for traumatized refugees. However, the type of supportive counseling applied in this study might not be representative of counseling practices in other organizations, as talking about the traumatic event was avoided in our supportive counseling condition. More research about the efficacy of different types of counseling is needed to specify effective mechanisms of this approach and to determine the conditions and duration of treatment that is necessary to achieve positive results.

Some limitations of the study indicate an urgent need for further studies in this field. The duration of treatment sessions was $90 \mathrm{~min}$ but could be extended up to $120 \mathrm{~min}$ for single exceptional sessions. Although extended sessions were scarce (about 10\%) and occurred in all conditions, we did not monitor the exact duration of each treatment and cannot fully rule out a dosage effect.

In spite of the promising treatment effect found in this trial, this study does not fully prove the usefulness of any psychotherapeutic approach for war-torn populations, as the treatment was carried out by well-trained European psychologists. The procedure is costly, and thus cannot open the way to large-scale interventions for the sizeable refugee populations that exist. Future dissemination studies should examine possible interventions that can utilize local paramedical staff who can be trained to implement trauma-focused treatments for PTSD. The pragmatic procedure of narrative exposure therapy should be feasible for local staff without any psychosocial background.

Furthermore, the issue of the high prevalence of comorbid symptoms that remained for all groups following treatment must be investigated. It is unclear whether normal levels of anxiety and depression can be achieved by any psychosocial treatment as long as the refugees remain in unsafe living conditions. Further studies should examine whether longer treatment approaches that extend the duration of exposure would be more effective. Another possibility would be to follow narrative exposure therapy with a shortterm, symptom-oriented treatment to improve symptoms of depression and anxiety.
The possible social and political implications of a narrative approach such as narrative exposure therapy require further examination. Finding ways to effectively reduce, as well as prevent, the psychological suffering of victims of war is an important challenge for scientists and aid organizations and indeed remains an ethical obligation. Bringing forth the narratives of victims may not only benefit the survivors but may also increase public awareness of severe human rights violations in dictatorships and war areas, as well as the public's ability and responsibility to make changes at both global and local levels. We maintain that the tradition of joint psychological and political approaches may offer opportunities to remedy mental suffering and to provide assistance to those in need in war-torn populations.

\section{References}

Amani Trust. (1997). A trauma counselling handbook. Harare, Zimbabwe: Author.

American Psychiatric Association. (1994). Diagnostic and statistical manual of mental disorders (4th ed.). Washington, DC: Author.

Bower, P., Rowland, N., Mellor, C., Heywood, P., Godfrey, C., \& Hardy, R. (2002). Effectiveness and cost effectiveness of counselling in primary care. Retrieved from Cochrane Database of Systematic Reviews, CD001025(1).

Cienfuegos, J., \& Monelli, C. (1983). The testimony of political repression as a therapeutic instrument. American Journal of Orthopsychiatry, 53, 43-51.

de Jong, J. T., Komproe, I. H., Van Ommeren, M., El Masri, M., Araya, M., Khaled, N., et al. (2001). Lifetime events and posttraumatic stress disorder in 4 postconflict settings. JAMA, 286, 555-562.

Ehlers, A., \& Clark, D. M. (2000). A cognitive model of posttraumatic stress disorder. Behavioral Research and Therapy, 38, 319-345.

Foa, E. B. (1995). Post-traumatic Stress Diagnostic Scale (PDS). Minneapolis, MN: National Computer Systems.

Foa, E. B. (2000). Psychosocial treatment of posttraumatic stress disorder. Journal of Clinical Psychiatry, 61(Suppl. 5), 43-48, 49-51.

Foa, E. B., Dancu, C. V., Hembree, E. A., Jaycox, L. H., Meadows, E. A., \& Street, G. P. (1999). A comparison of exposure therapy, stress inoculation training, and their combination for reducing posttraumatic stress disorder in female assault victims. Journal of Consulting and Clinical Psychology, 67, 194-200.

Foa, E. B., \& Rothbaum, B. O. (1998). Treating the trauma of rape: Cognitive-behavioral therapy for PTSD. New York: Guilford Press.

Foa, E. B., Rothbaum, B. O., Riggs, D. S., \& Murdock, T. B. (1991). Treatment of posttraumatic stress disorder in rape victims: A comparison between cognitive-behavioral procedures and counseling. Journal of Consulting and Clinical Psychology, 59, 715-723.

Harding, T. W., de Arango, M. V., Baltazar, J., Climent, C. E., Ibrahim, H. H., Ladrido-Ignacio, L., et al. (1980). Mental disorders in primary health care: A study of their frequency and diagnosis in four developing countries. Psychological Medicine, 10, 231-241.

Hollifield, M., Warner, T. D., Lian, N., Krakow, B., Jenkins, J. H., Kesler, J., et al. (2002). Measuring trauma and health status in refugees: A critical review. JAMA, 288, 611-621.

Karunakara, U., Neuner, F., Schauer, M., Singh, K., Hill, K., Elbert, T., \& Burnham, G. (in press). Traumatic events and symptoms of posttraumatic stress disorder amongst Sudanese nationals, refugees, and Ugandan nationals in the West Nile. African Health Sciences.

Marks, I., Lovell, K., Noshirvani, H., Livanou, M., \& Thrasher, S. (1998) Treatment of posttraumatic stress disorder by exposure and/or cognitive restructuring: A controlled study. Archives of General Psychiatry, 55, 317-325.

Maslow, A. H. (1943). A theory of human motivation. Psychological Review, 50, 370-396. 
Mollica, R. F., Donelan, K., Tor, S., Lavelle, J., Elias, C., Frankel, M., et al. (1993). The effect of trauma and confinement on functional health and mental health status of Cambodians living in Thailand-Cambodia border camps. JAMA, 270, 581-586.

Neuner, F., Schauer, M., Elbert, T., \& Roth, W. T. (2002). A narrative exposure treatment as intervention in a refugee camp: A case report. Journal of Behavioural and Cognitive Psychotherapy, 30, 205-209.

Paunovic, N., \& Ost, L. G. (2001). Cognitive-behavior therapy vs exposure therapy in the treatment of PTSD in refugees. Behavioral Research and Therapy, 39, 1183-1197.

Schauer, M., Karunakara, U., Neuner, F., Klaschik, C., Kley, E., Rockstroh, B., et al. (2001, May). High prevalence of PTSD in African victims of organized violence: A demographic survey in the West Nile region of Uganda and Sudan. Paper presented at the 7th European Conference on Traumatic Stress Studies, Edinburgh, Scotland, United Kingdom.

Schauer, M., Neuner, F., \& Elbert, T. (in press). The victim's voice: Manual of narrative exposure therapy for the treatment of survivors of war and torture. Göttingen, Germany: Hogrefe \& Huber.

Tarrier, N., Pilgrim, H., Sommerfield, C., Faragher, B., Reynolds, M., Graham, E., et al. (1999). A randomized trial of cognitive therapy and imaginal exposure in the treatment of chronic posttraumatic stress disorder. Journal of Consulting and Clinical Psychology, 67, 13-18.

Van der Veer, G. (1998). Counselling and therapy with refugees and victims of trauma (Vol. 2). West Sussex: Wiley.

Ware, J., Jr., Kosinski, M., \& Keller, S. D. (1996). A 12-Item Short-Form Health Survey: Construction of scales and preliminary tests of reliability and validity. Medical Care, 34, 220-233.

Weine, S. M., Kulenovic, A. D., Pavkovic, I., \& Gibbons, R. (1998). Testimony psychotherapy in Bosnian refugees: A pilot study. American Journal of Psychiatry, 155, 1720-1726.

World Health Organization. (1997). Composite International Diagnostic Interview (CIDI). Geneva, Switzerland: Author.

World Health Organization/United Nations High Commissioner for Refugees. (1996). Mental health of refugees. Geneva: World Health Organization. 